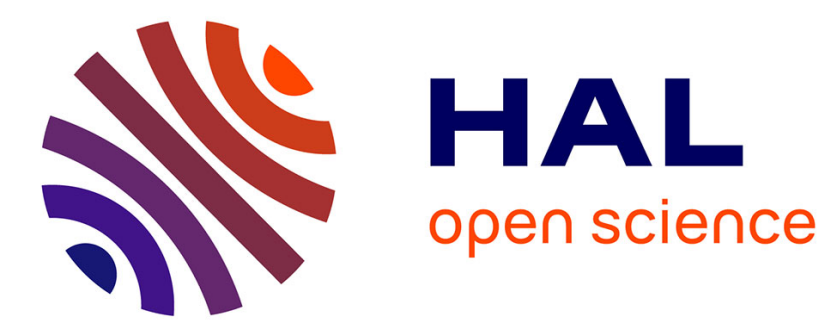

\title{
Fixed-time sliding mode control with mismatched disturbances
}

Emmanuel Moulay, Vincent Léchappé, Emmanuel Bernuau, Michael Defoort, Franck Plestan

\section{- To cite this version:}

Emmanuel Moulay, Vincent Léchappé, Emmanuel Bernuau, Michael Defoort, Franck Plestan. Fixedtime sliding mode control with mismatched disturbances. Automatica, 2022, 136, pp.110009. 10.1016/j.automatica.2021.110009 . hal-03502587

\section{HAL Id: hal-03502587 \\ https://hal.science/hal-03502587}

Submitted on 25 Dec 2021

HAL is a multi-disciplinary open access archive for the deposit and dissemination of scientific research documents, whether they are published or not. The documents may come from teaching and research institutions in France or abroad, or from public or private research centers.
L'archive ouverte pluridisciplinaire HAL, est destinée au dépôt et à la diffusion de documents scientifiques de niveau recherche, publiés ou non, émanant des établissements d'enseignement et de recherche français ou étrangers, des laboratoires publics ou privés. 


\title{
Fixed-time sliding mode control with mismatched disturbances
}

\author{
Emmanuel Moulay ${ }^{a}$, Vincent Léchappé $^{\mathrm{b}}$, Emmanuel Bernuau ${ }^{\mathrm{c}}$, Michael Defoort $^{\mathrm{d}}$, \\ Franck Plestan ${ }^{\mathrm{e}}$ \\ ${ }^{\mathrm{a}}$ XLIM (UMR CNRS 7252), Université de Poitiers, 11 bd Marie et Pierre Curie, 86073 Poitiers Cedex 9, France \\ ${ }^{\mathrm{b}}$ Laboratoire Ampère (UMR CNRS 5005), INSA de Lyon, 20 Avenue Albert Einstein, 69100 Villeurbanne, France \\ ${ }^{\mathrm{c}}$ INRAE, AgroParisTech, UMR 1145 Joint Research Unit for Food Process Engineering, 91300, Massy, France \\ ${ }^{\mathrm{d}}$ LAMIH (UMR CNRS 8201), INSA Hauts-de-France, Polytechnic University Hauts-de-France, 59313 Valenciennes, France \\ ${ }^{\mathrm{e}}$ LS2N (UMR CNRS 6004), École Centrale de Nantes, 1 Rue de la Noë, 44321 Nantes Cedex 3, France
}

\begin{abstract}
The aim of this article is to develop new second-order fixed-time sliding mode controllers able to tackle mismatched disturbances. Contrary to what is done in the literature, the proposed sliding-mode control strategy does not use disturbance observers because the sliding surface possesses robustness properties. The results are applied to define a new fixed-time differentiator.
\end{abstract}

Key words: Sliding mode control, fixed-time stability, mismatched disturbances, fixed-time differentiator

\section{Introduction}

Sliding mode control (SMC) has been developed since the seminal work of Utkin [1] in the 70s, see for instance [2] for more details. Recently, the problem of robust fixed-time SMC was treated in [3,4] where fixed-time stability developed in [5-7] ensures that all solutions converge to the equilibrium point in a fixed-time. However, the proposed controllers were rather complex to implement due to a problem of singularity avoidance coming from the fixed-time stability strategy. In [8], simple robust controllers are proposed by using state-dependent variable exponent coefficient in the sliding surfaces and the controllers.

All the above mentioned articles deal with matched disturbances which are disturbances acting on the control input channel and are the most common disturbances encountered in control applications. Mismatched disturbances acting on other channels than the control channel also appear in automatic control for instance in [9-23]

Email addresses: emmanuel.moulay@univ-poitiers.fr (Emmanuel Moulay), vincent.lechappe@insa-lyon.fr (Vincent Léchappé), emmanuel . bernuau@agroparistech.fr (Emmanuel Bernuau), michael.defoort@uphf.fr (Michael Defoort), franck.plestan@ec-nantes.fr (Franck Plestan). and it can model the DC-DC buck power converter systems [24]. In the previous works, the control problems are solved by using observers to estimate mismatched disturbances. In our article, we use a fixed-time SMC strategy adapted from [8] which is known to be robust in order to reject the mismatched disturbance of a secondorder system without using disturbance observers. This renders this new strategy easier to tune. To the best of the authors' knowledge, this is the first time that a mismatched disturbance is rejected by using a fixed-time sliding surface known for its robustness properties. Thus, we adapt the sliding-mode controller developed in [8] in order to be used with a mismatched disturbance. The results are tested in simulation on the double integrator in order to show the efficiency of the proposed method. Finally, the proposed control strategy is applied to develop a new fixed-time differentiator, the reader may refer to [25] for more details on differentiators.

The article is organized as follows. After some preliminaries given in Section 2, the main results are presented in Section 3 and tested on the double integrator with matched and mismatched disturbances. They are also applied to develop a new fixed-time differentiator. Finally, a conclusion is addressed in Section 4. 


\section{Preliminaries}

In the following, denote $e$ the constant such that $\ln (e)=$ 1. Let us recall some results on finite-time stability and fixed-time stability. Consider the following ordinary differential equation

$$
\begin{aligned}
& \dot{x}(t)=f(x(t)), \quad x(t) \in \mathbb{R}^{n} \\
& x(0)=x_{0}
\end{aligned}
$$

with $f$ a locally essentially bounded function.

Definition 1 [26,27] The origin is globally finite-time stable for system (1) if it is Lyapunov stable and for all $x_{0} \in \mathbb{R}^{n}$ there exists $T\left(x_{0}\right) \geqslant 0$ such that, for any $x(\cdot)$ solution of (1) with $x(0)=x_{0}, \lim _{t \rightarrow T\left(x_{0}\right)}\|x(t)\|=0$, i.e. $\|x(t)\| \equiv 0$ for all $t \geqslant T\left(x_{0}\right)$. Such a function $T$ is called a settling-time function.

Definition 2 [6] System (1) is globally fixed-time stable if:

(1) it is globally finite-time stable;

(2) there exists a globally upper bounded settling-time function $T$, i.e. there exists a constant $\mathbf{T}>0$, independent of the initial condition, such that for all $x_{0} \in \mathbb{R}^{n}$, $T\left(x_{0}\right) \leqslant \mathbf{T}$.

\section{Main results}

Consider the following uncertain nonlinear second-order system

$$
\left\{\begin{array}{l}
\dot{x}_{1}=x_{2}+d_{1} \\
\dot{x}_{2}=f(x)+g(x) u+d_{2}
\end{array}\right.
$$

with $x=\left(x_{1}, x_{2}\right) \in \mathbb{R}^{2}$ the state, $u \in \mathbb{R}$ the control input, $f$ and $g$ continuous functions such that $f(0)=0, g(x) \neq$ 0 for all $x \in \mathbb{R}^{2}, d_{1}$ a mismatched disturbance such that $\left|d_{1}(t)\right|<\delta_{1}$ and $d_{2}$ a matched disturbance such that $\left|d_{2}(t)\right|<\delta_{2}$. The equilibrium trajectory of system $(2)$ is given by $\left(0,-d_{1}(t)\right)$. The second order systems with mismatched disturbances have been studied for instance in $[19]$.

The main objective of this subsection is to design a sliding variable leading to global robust fixed-time stabilization of system (2) towards the equilibrium trajectory. Consider the sliding variable with a state-dependent variable exponent coefficient given by

$$
s(x)=x_{2}+\beta\left|x_{1}\right|^{\frac{\lambda_{1} x_{1}^{2}}{1+\mu_{1} x_{1}^{2}}} \operatorname{sgn}\left(x_{1}\right)
$$

with $\lambda_{1}>0, \mu_{1}>0$ such that $\theta_{1}=\frac{\lambda_{1}}{1+\mu_{1}}>1, \beta>\delta_{1} e^{\frac{\lambda_{1}}{2 e}}$ and the following new controller inspired from Proposi- tion 2 in [8]

$$
\begin{aligned}
u(x)=-g(x)^{-1}[f(x) & +k_{1} \operatorname{sgn}(s)|s|^{\frac{\lambda_{2} s^{2}}{1+\mu_{2} s^{2}}} \\
& \left.+\varphi\left(\left|x_{1}\right|\right)\left(x_{2}+k_{2} \operatorname{sgn}(s)\right)\right]
\end{aligned}
$$

with $\lambda_{2}>0, \mu_{2}>0$ such that $\theta_{2}=\frac{\lambda_{2}}{1+\mu_{2}}>1$ and

$$
\varphi(z)=\frac{\beta \lambda_{1}}{1+\mu_{1} z^{2}}\left(\frac{2 \ln (z)}{1+\mu_{1} z^{2}}+1\right) z^{\frac{\lambda_{1} z^{2}}{1+\mu_{1} z^{2}}+1} .
$$

Let us state a first technical lemma.

Lemma 1 For $z \geqslant 0$, we have $\varphi(z)>0$ when $z>1$ and $|\varphi(z)| \leqslant 2 \beta \lambda_{1}\left(1+\mu_{1}\right)$ when $0 \leqslant z \leqslant 1$.

Proof. The first statement is obvious. When $0 \leqslant z \leqslant 1$, we write

$$
\varphi(z)=\frac{\beta \lambda_{1}}{\left(1+\mu_{1} z^{2}\right)^{2}}\left(2 z \ln (z)+z+\mu_{1} z^{3}\right) z^{\frac{\lambda_{1} z^{2}}{1+\mu_{1} z^{2}}} .
$$

We see that $0 \leqslant \frac{\beta \lambda_{1}}{\left(1+\mu_{1} z^{2}\right)^{2}} \leqslant \beta \lambda_{1}$ and $0 \leqslant z^{\frac{\lambda_{1} z^{2}}{1+\mu_{1} z^{2}}} \leqslant 1$. Now denoting

$$
\psi(z)=2 z \ln (z)+z+\mu_{1} z^{3}
$$

we see that $\psi^{\prime}(z)=2 \ln (z)+3+3 \mu_{1} z^{2}$. Denoting $z_{0}$ the unique solution of $\psi^{\prime}(z)=0$, we see that $\psi$ is decreasing on $\left(0, z_{0}\right)$ and increasing on $\left(z_{0}, 1\right)$. Therefore it leads to $|\psi(z)| \leqslant \max \left\{1+\mu_{1},\left|\psi\left(z_{0}\right)\right|\right\}$. But, given that $\psi^{\prime}\left(z_{0}\right)=$ 0 , we have

$$
\begin{aligned}
\psi\left(z_{0}\right) & =z_{0}\left(2 \ln \left(z_{0}\right)+1+\mu_{1} z_{0}^{2}\right) \\
& =z_{0}\left(-3-3 \mu_{1} z_{0}^{2}+1+\mu_{1} z_{0}^{2}\right) \\
& =-2 z_{0}\left(1+\mu_{1} z_{0}\right)
\end{aligned}
$$

Finally, because $0 \leqslant z_{0} \leqslant 1$, we see that $\left|\psi\left(z_{0}\right)\right| \leqslant 2(1+$ $\left.\mu_{1}\right)$ and we get the result.

We can now state the first theorem.

Theorem 1 If $k_{1}>\delta_{3}=\delta_{2}+2 \beta \lambda_{1}\left(1+\mu_{1}\right)\left(\delta_{1}+k_{2}\right)$ and $k_{2}>\delta_{1}$, the closed-loop system $(2)-(3)-(4)$ reaches the equilibrium trajectory $\left(0,-d_{1}(t)\right)$ in a fixed time satisfying

$$
\begin{aligned}
T\left(x_{0}\right) \leqslant & \frac{1}{\left(k_{1}-\delta_{3}\right)\left(\theta_{2}-1\right)}+\frac{1}{k_{1} e^{\frac{-\lambda_{2}}{2 e}}-\delta_{3}} \\
& +\frac{1}{\left(\beta-\delta_{1}\right)\left(\theta_{1}-1\right)}+\frac{1}{\beta e^{\frac{-\lambda_{1}}{2 e}}-\delta_{1}} .
\end{aligned}
$$


Proof. One has

$$
\begin{aligned}
\dot{s}= & f(x)+g(x) u(x) \\
& +\frac{\beta \lambda_{1}\left|x_{1}\right|\left(x_{2}+d_{1}\right)}{1+\mu_{1} x_{1}^{2}}\left(\frac{2 \ln \left|x_{1}\right|}{1+\mu_{1} x_{1}^{2}}+1\right)\left|x_{1}\right|^{\frac{\lambda_{1} x_{1}^{2}}{1+\mu_{1} x_{1}^{2}}}+d_{2} \\
= & -k_{1}|s|^{\frac{\lambda_{2} s^{2}}{1+\mu_{2} s^{2}}} \operatorname{sgn}(s)+d_{2}+\varphi\left(\left|x_{1}\right|\right)\left(d_{1}-k_{2} \operatorname{sgn}(s)\right)
\end{aligned}
$$

Now, considering the Lyapunov function $V(s)=s^{2}$, we see that

$$
\dot{V}(s) \leqslant 2|s|\left(-k_{1}|s|^{\frac{\lambda_{2} s^{2}}{1+\mu_{2} s^{2}}}+\delta_{2}+\varphi\left(\left|x_{1}\right|\right)\left(\operatorname{sgn}(s) d_{1}-k_{2}\right)\right) .
$$

When $\left|x_{1}\right|>1$, we have $\varphi\left(\left|x_{1}\right|\right)>0$, therefore

$$
\dot{V}(s) \leqslant 2|s|\left(-k_{1}|s|^{\frac{\lambda_{2} s^{2}}{1+\mu_{2} s^{2}}}+\delta_{2}+\varphi\left(\left|x_{1}\right|\right)\left(\delta_{1}-k_{2}\right)\right) .
$$

But given that $k_{2}>\delta_{1}$, we get $\varphi\left(\left|x_{1}\right|\right)\left(\delta_{1}-k_{2}\right)<0$ which in turn yields

$$
\dot{V}(s) \leqslant 2|s|\left(-k_{1}|s|^{\frac{\lambda_{2} s^{2}}{1+\mu_{2} s^{2}}}+\delta_{2}\right) \text {. }
$$

When $\left|x_{1}\right| \leqslant 1$, we have $\left|\varphi\left(\left|x_{1}\right|\right)\right| \leqslant 2 \beta \lambda_{1}\left(1+\mu_{1}\right)$ and thus

$$
\dot{V}(s) \leqslant 2|s|\left(-k_{1}|s|^{\frac{\lambda_{2} s^{2}}{1+\mu_{2} s^{2}}}+\delta_{2}+2 \beta \lambda\left(1+\mu_{1}\right)\left(\delta_{1}+k_{2}\right)\right) \text {. }
$$

Finally, we see that

$$
\dot{V}(s) \leqslant 2|s|\left(-k_{1}|s|^{\frac{\lambda_{2} s^{2}}{1+\mu_{2} s^{2}}}+\delta_{3}\right)
$$

where $\delta_{3}=\delta_{2}+2 \beta \lambda_{1}\left(1+\mu_{1}\right)\left(\delta_{1}+k_{2}\right)$. By using Theorem 2 in [8], one deduces that system (6) starting at $s(0)=s_{0}$ reaches the sliding surface $\{s=0\}$ in a fixedtime satisfying $T\left(s_{0}\right) \leqslant \frac{1}{\left(k_{1}-\delta_{3}\right)\left(\theta_{2}-1\right)}+\frac{1}{k_{1} e^{\frac{-\lambda_{2}}{2 e}}-\delta_{3}}$. From (3), one has

$$
\dot{x}_{1}=-\beta\left|x_{1}\right|^{\frac{\lambda_{1} x_{1}^{2}}{1+\mu_{1} x_{1}^{2}}} \operatorname{sgn}\left(x_{1}\right)+d_{1} \text {. }
$$

By using one more time Theorem 2 in [8], it is deduced that $x_{1}(t)$ starting at $x_{1}(0)=x_{10}$ reaches the origin in a fixed-time satisfying $T\left(x_{10}\right) \leqslant \frac{1}{\left(\beta-\delta_{1}\right)\left(\theta_{1}-1\right)}+\frac{1}{\beta e^{\frac{-\lambda_{1}}{2 e}}-\delta_{1}}$. Finally, the closed-loop system $(2)-(3)-(4)$ reaches the equilibrium trajectory $\left(0,-d_{1}(t)\right)$ in a fixed time satisfying $T\left(x_{0}\right)=T\left(s_{0}\right)+T\left(x_{10}\right)$.
Remark 1 Let us remark that if we want to use the controller given in Proposition 2 of [8]

$$
\begin{aligned}
u(x)= & -g(x)^{-1}\left[f(x)+k|s|^{\frac{\lambda_{2} s^{2}}{1+\mu_{2} s^{2}}} \operatorname{sgn}(s)\right. \\
& \left.+\frac{\beta \lambda_{1}\left|x_{1}\right| x_{2}}{1+\mu_{1} x_{1}^{2}}\left(\frac{2 \ln \left|x_{1}\right|}{1+\mu_{1} x_{1}^{2}}+1\right)\left|x_{1}\right|^{\frac{\lambda_{1} x_{1}^{2}}{1+\mu_{1} x_{1}^{2}}}\right]
\end{aligned}
$$

with $\theta_{2}=\frac{\lambda_{2}}{1+\mu_{2}}>1, k>\delta_{2} e^{\frac{\lambda_{2}}{2 e}}$ then we have to prove that $\frac{\beta \lambda_{1}\left|x_{1}\right| d_{1}}{1+\mu_{1} x_{1}^{2}}\left(\frac{2 \ln \left|x_{1}\right|}{1+\mu_{1} x_{1}^{2}}+1\right)\left|x_{1}\right|^{\frac{\lambda_{1} x_{1}^{2}}{1+\mu_{1} x_{1}^{2}}}$ is bounded, i.e.

$$
\lim _{x_{1} \rightarrow \infty} \frac{\beta \lambda_{1}\left|x_{1}\right| d_{1}}{1+\mu_{1} x_{1}^{2}}\left(\frac{2 \ln \left|x_{1}\right|}{1+\mu_{1} x_{1}^{2}}+1\right)\left|x_{1}\right|^{\frac{\lambda_{1} x_{1}^{2}}{1+\mu_{1} x_{1}^{2}}}<+\infty .
$$

It leads to the condition $\lambda_{1} \leqslant \mu_{1}$ because $\left|x_{1}\right|^{\frac{\lambda_{1} x_{1}^{2}}{1+\mu_{1} x_{1}^{2}}}$ behaves as $\left|x_{1}\right|^{\frac{\lambda}{\mu}}$ far from the origin $x_{1}=0$. However, we have the condition $1+\mu_{1}<\lambda_{1}$ coming from Theorem 2 in [8] which is incompatible with the condition $\lambda_{1} \leqslant \mu_{1}$. This is the reason why we developed the new controller (4) which is discontinuous in the sliding variable but able to manage the mismatched disturbance.

Remark 2 With Theorem 1, we obtain that $x_{2}(t)$ tracks $-d_{1}(t)$ in a fixed time. Thus, we obtain a new fixed-time estimator of the mismatched disturbance without using observers.

Consider now the following new controller inspired from Proposition 3 in [8]

$$
\begin{array}{r}
u(x)=-g(x)^{-1}\left[f(x)+k_{1} \operatorname{sgn}(s)+k_{2}|s|^{\alpha} \operatorname{sgn}(s)\right. \\
\left.+\varphi\left(\left|x_{1}\right|\right)\left(x_{2}+k_{3} \operatorname{sgn}(s)\right)\right]
\end{array}
$$

with $\alpha>1$

Theorem 2 If $k_{1}>\delta_{3}=\delta_{2}+2 \beta \lambda_{1}\left(1+\mu_{1}\right)\left(\delta_{1}+k_{3}\right)$, $k_{2}>\delta_{3}$ and $k_{3}>\delta_{1}$ then the closed-loop system (2)(3)-(15) reaches the equilibrium trajectory $\left(0,-d_{1}(t)\right)$ in a fixed time satisfying

$$
\begin{aligned}
T\left(x_{0}\right) \leqslant & \frac{1}{\left(k_{2}-\delta_{3}\right)(\alpha-1)}+\frac{1}{k_{1}-\delta_{3}} \\
& +\frac{1}{\left(\beta-\delta_{1}\right)\left(\theta_{1}-1\right)}+\frac{1}{\beta e^{\frac{-\lambda_{1}}{2 e}}-\delta_{1}} .
\end{aligned}
$$

Proof. Similarly to the proof of Theorem 1, we get, with $V(s)=s^{2}$ :

$$
\dot{V}(s) \leqslant 2|s|\left(-k_{1}-k_{2}|s|^{\alpha}+\delta_{3}\right)
$$


where $\delta_{3}=\delta_{2}+2 \beta \lambda_{1}\left(1+\mu_{1}\right)\left(\delta_{1}+k_{3}\right)$. If $|s|>1$, we see that $\dot{V} \leqslant 2|s|\left(\delta_{3}-k_{2}|s|^{\alpha}\right)$. But given that $|s|>1$ and $\delta_{3}>0$, we get

$$
\begin{aligned}
\dot{V}(s) & \leqslant 2|s|\left(\delta_{3}|s|^{\alpha}-k_{2}|s|^{\alpha}\right) \\
& \leqslant 2\left(\delta_{3}-k_{2}\right) V(s)^{\frac{1+\alpha}{2}}
\end{aligned}
$$

which yields, as long as $|s(t)|>1,1 \geqslant V(s(t))^{\frac{1-\alpha}{2}} \geqslant$ $(1-\alpha)\left(\delta_{3}-k_{2}\right) t$. This inequality shows that $s(t)$ reaches the set $\{V \leqslant 1\}$ in a time $T_{1} \leqslant \frac{1}{(1-\alpha)\left(\delta_{3}-k_{2}\right)}$. If $|s| \leqslant 1$ we get

$$
\dot{V}(s) \leqslant 2|s|\left(\delta_{3}-k_{1}\right) \leqslant 2\left(\delta_{3}-k_{1}\right) V^{\frac{1}{2}} .
$$

Therefore, as long as $s(t)>0, V(s(t))^{\frac{1}{2}} \leqslant 1+\left(\delta_{3}-\right.$ $\left.k_{1}\right) t$, which yields that $s(t)$ reaches 0 in a time $T_{2} \leqslant$ $\frac{1}{k_{1}-\delta_{3}}$. Finally, we conclude that, regardless of its initial condition, $s(t)$ reaches 0 in a fixed-time $T\left(s_{0}\right) \leqslant T_{1}+T_{2}$. The end of the proof is similar to the one of Theorem 1.

Some guidelines are provided below in order to tune the parameters of controller (15):

1. choose $\delta_{1}$ and $\delta_{2}$ according to the maximum values of disturbances $d_{1}$ and $d_{2}$;

2. take $\lambda_{1}>0$ and $\mu_{1}>0$ such that $\theta_{1}=\frac{\lambda_{1}}{1+\mu_{1}}>1$;

3. take $\beta$ such that $\beta>\delta_{1} e^{\frac{\lambda_{1}}{2 e}}$;

4. take $k_{3}>\delta_{1} ; 5$. take $k_{1}>\delta_{3}$ and $k_{2}>\delta_{3}$ with $\delta_{3}=\delta_{2}+2 \beta \lambda_{1}\left(1+\mu_{1}\right)\left(\delta_{1}+k_{3}\right)$;

6 . take $\alpha>1$.

The guidelines for the tuning of controller (4) can be obtained in a similar way.

Example 1 The results of Theorem 1 and Theorem 2 have been tested in simulation on a double integrator, i.e. taking $f=0$ and $g=1$ in system (2). The following disturbances have been chosen $d_{1}(t)=\sin (5 t)$ and $d_{2}(t)=2 \sin (10 t)$ so $\delta_{1}=1$ and $\delta_{2}=2$. The Euler solver with a fixed step of $10^{-4} \mathrm{~s}$ has been used. The parameters of both controllers are as follows: $\lambda_{1}=3, \mu_{1}=1, \beta=5.5$, $k_{1}=30, \lambda_{2}=4, \mu_{2}=1, k_{2}=2$ for controller (4) and $\lambda_{1}=3, \mu_{1}=1, \beta=5.5, k_{1}=39, k_{2}=20, k_{3}=2, \alpha=3$ for controller (15). This set of parameters verifies the conditions of Theorem 1 and Theorem 2. The results of the simulations with the initial condition $x(0)=[1,3]^{T}$ are displayed on Figure 1(a)-(b). For comparison purpose, one has implemented the fixed-time controller given in [6]. One can observe that after a reaching phase, the state $x_{1}$ converges to 0 . Note that the dynamics of $x_{2}$ tracks the unknown mismatched disturbance $-d_{1}$. The upper bound of the settling-time (5) given in Theorem 1 is equal to 30s and the upper bound of the settling-time (16) given in Theorem 2 is equal to 8.5s. One can see that this settling-time is much smaller in the simulation, around $1 \mathrm{~s}$ for controller (4) and around $2 \mathrm{~s}$ for controller (15). Let us notice that the fixed-time controller for the second order system proposed in [6] and displayed on Figure 1(c) is
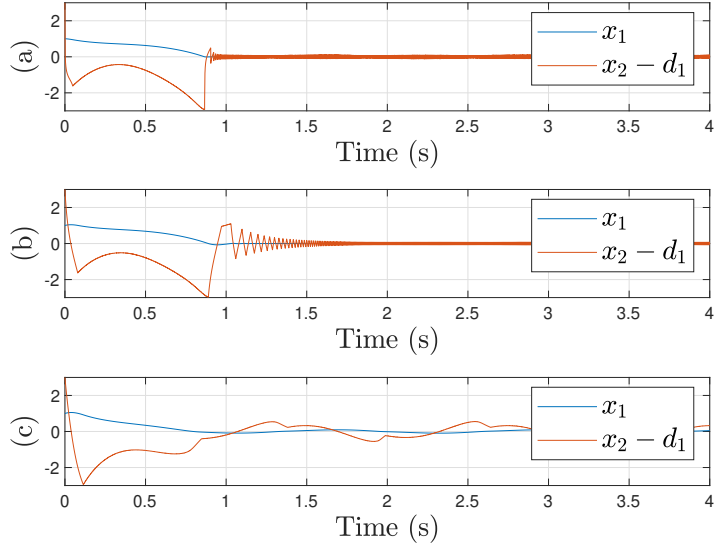

Fig. 1. (a) Double integrator with sliding surface (3) and controller (4); (b) Double integrator with sliding surface (3) and controller (15); (c) Double integrator with controller [6].

not able to manage the mismatched disturbances $d_{1}$, contrary to the proposed controllers (4) and (15) displayed on Figures 1(a)-(b) respectively.

Example 2 Let us consider a differentiable signal $h(t)$ defined on $[0, \infty)$ which is differentiable with bounded time derivative, i.e. $|\dot{h}(t)| \leqslant \delta_{1}$. The objective here is to estimate in fixed-time the time derivative of this signal. To achieve this objective, let us consider the following fixed-time differentiator:

$$
\begin{aligned}
& \dot{\hat{x}}_{1}=\hat{x}_{2} \\
& \dot{\hat{x}}_{2}=u\left(\hat{x}_{1}-h, \hat{x}_{2}\right)
\end{aligned}
$$

where $u\left(\hat{x}_{1}-h, \hat{x}_{2}\right)$ is defined in (4) or (15) with $f=0$ and $g=1$. Defining the error $e=\hat{x}_{1}-h$, one obtains

$$
\begin{aligned}
\dot{e} & =\hat{x}_{2}-\dot{h} \\
\dot{\hat{x}}_{2} & =u\left(e, \hat{x}_{2}\right)
\end{aligned}
$$

It yields system (2) with $d_{2}=0$ and $d_{1}=-\dot{h}$. In the following simulations, the signal has been chosen as $h(t)=0.2 \cos \left(\frac{1}{5} t\right)$ so $\delta_{1}=1$ and $\delta_{2}=0$. The Euler solver with a fixed step of $10^{-5} \mathrm{~s}$ has been used. The parameters of $u$ are as follows: $\lambda_{1}=2.5, \mu_{1}=1, \beta=2, k_{1}=150$, $\lambda_{2}=2.2, \mu_{2}=1, k_{2}=3$ for scheme (4) and $\lambda_{1}=2.5$, $\mu_{1}=1, \beta=2, k_{1}=150, k_{2}=45, k_{3}=1.1, \alpha=3$ for scheme (15). This set of parameters verifies the conditions of Theorem 1 and Theorem 2. Furthermore, an additional uniformly bounded random measurement noise of magnitude $10^{-2}$ is considered in the following simulations. The results of the simulations with the initial condition $\hat{x}(0)=[1,1]^{T}$ are displayed on Figure 2 and 3. One can observe that after a reaching phase, the state $\hat{x}_{1}$ converges to $h$. Note that the dynamics of $\hat{x}_{2}$ converges to 

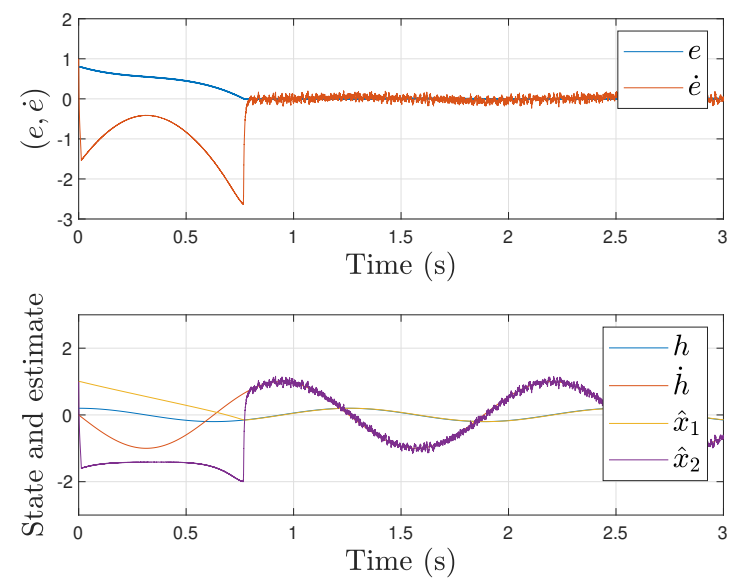

Fig. 2. (a) Fixed-time observer with sliding surface (3) and scheme (4).
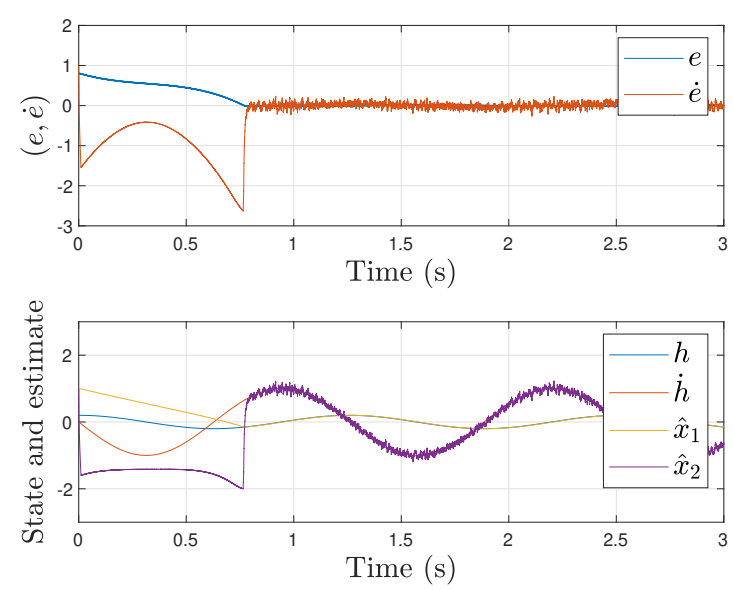

Fig. 3. Fixed-time observer with sliding surface (3) and scheme (15)

$\dot{h}$ in fixed time. The upper bound of the settling-time (5) given in Theorem 1 is equal to 8s and the upper bound of the settling-time (16) given in Theorem 2 is equal to 8s. One can see that this settling-time is much smaller in the simulation, around $0.9 \mathrm{~s}$ for both schemes.

It is worth mentioning that there exist several finite time differentiators (see for instance [28]). Nevertheless, the convergence time, grows unboundedly with respect to the initial differentiation error. Recently, an arbitrary order fixed-time differentiator, which can been seen as a generalization of the Levant's differentiator, has been introduced in [29]. For comparison purpose, the root mean square error (RMSE) after the reaching phase for our proposed differentiator (22) and [29] is as follows: $2.84 e-$ 05 when using (22) with u given in Theorem 1, 3.06e- 05 when using (22) with $u$ given in Theorem 2, 4.72e- 05 when uisng [29]. It can be seen that our proposed differentiators achieve better accuracy in the presence of measurement noise than the first order fixed-time differen- tiator proposed in [29].

\section{Conclusion}

A new sliding mode controller is proposed in order to get fixed time-convergence of second order systems in presence of unknown but bounded matched and mismatched perturbations. It is applied to develop a new fixed-time differentiator. The fixed-time controller is based on the design of a robust sliding surface and does not require any disturbance observer which simplifies the solution. For future works, a high order SMC or implicit SMC strategy could be used for reducing the chattering when dealing with robust fixed-time stabilization.

\section{References}

[1] V. Utkin, Variable structure systems with sliding modes, IEEE Transactions on Automatic Control 22 (2) (1977) 212222 .

[2] Y. Shtessel, C. Edwards, L. Fridman, A. Levant, Sliding mode control and observation, Control Engineering, Birkhäuser, 2014.

[3] M. L. Corradini, A. Cristofaro, Nonsingular terminal slidingmode control of nonlinear planar systems with global fixedtime stability guarantees, Automatica 95 (2018) 561-565.

[4] Z. Zuo, Non-singular fixed-time terminal sliding mode control of non-linear systems, IET Control Theory \& Applications 9 (4) (2015) 545-552.

[5] V. Andrieu, L. Praly, A. Astolfi, Homogeneous approximation, recursive observer design and output feedback, SIAM Journal on Control and Optimization 47 (4) (2008) 1814-1850.

[6] A. Polyakov, Nonlinear feedback design for fixed-time stabilization of linear control systems, IEEE Transactions on Automatic Control 57 (8) (2012) 2106-2110.

[7] A. Polyakov, Generalized homogeneity in systems and control, Communications and Control Engineering, Springer, 2020.

[8] E. Moulay, V. Léchappé, E. Bernuau, F. Plestan, Robust fixed-time stability: application to sliding mode control, IEEE Transactions on Automatic Control (2021). doi:10.1109/TAC.2021.3069667.

[9] D. Ginoya, P. Shendge, S. Phadke, Sliding mode control for mismatched uncertain systems using an extended disturbance observer, IEEE Transactions on Industrial Electronics 61 (4) (2013) 1983-1992.

[10] X. Han, E. Fridman, S. K. Spurgeon, Sliding-mode control of uncertain systems in the presence of unmatched disturbances with applications, International Journal of Control 83 (12) (2010) 2413-2426.

[11] E. Kayacan, T. I. Fossen, Feedback linearization control for systems with mismatched uncertainties via disturbance observers, Asian Journal of Control 21 (3) (2019) 1064-1076.

[12] S. Li, J. Yang, W.-H. Chen, X. Chen, Generalized extended state observer based control for systems with mismatched uncertainties, IEEE Transactions on Industrial Electronics 59 (12) (2011) 4792-4802. 
[13] S. Li, H. Sun, J. Yang, X. Yu, Continuous finite-time output regulation for disturbed systems under mismatching condition, IEEE Transactions on Automatic Control 60 (1) (2015) 277-282.

[14] J. A. Moreno, M. Osorio, Strict lyapunov functions for the super-twisting algorithm, IEEE Transactions on Automatic Control 57 (4) (2012) 1035-1040.

[15] H. Sun, L. Guo, Composite adaptive disturbance observer based control and back-stepping method for nonlinear system with multiple mismatched disturbances, Journal of the Franklin Institute 351 (2) (2014) 1027-1041.

[16] H. Sun, S. Li, J. Yang, L. Guo, Non-linear disturbance observer-based back-stepping control for airbreathing hypersonic vehicles with mismatched disturbances, IET Control Theory \& Applications 8 (17) (2014) 1852-1865.

[17] B. Tian, H. Lu, Z. Zuo, H. Wang, Fixed-time stabilization of high-order integrator systems with mismatched disturbances, Nonlinear Dynamics 94 (4) (2018) 2889-2899.

[18] J. Yang, W.-H. Chen, S. Li, Non-linear disturbance observer-based robust control for systems with mismatched disturbances/uncertainties, IET Control Theory \& Applications 5 (18) (2011) 2053-2062.

[19] J. Yang, S. Li, J. Su, X. Yu, Continuous nonsingular terminal sliding mode control for systems with mismatched disturbances, Automatica 49 (7) (2013) 2287-2291.

[20] J. Zhang, X. Liu, Y. Xia, Z. Zuo, Y. Wang, Disturbance observer-based integral sliding-mode control for systems with mismatched disturbances, IEEE Transactions on Industrial Electronics 63 (11) (2016) 7040-7048.

[21] H. Zhang, X. Wei, L. Zhang, M. Tang, Disturbance rejection for nonlinear systems with mismatched disturbances based on disturbance observer, Journal of the Franklin Institute 354 (11) (2017) 4404-4424.

[22] C. Zhang, Y. Yan, C. Wen, J. Yang, H. Yu, A nonsmooth composite control design framework for nonlinear systems with mismatched disturbances: Algorithms and experimental tests, IEEE Transactions on Industrial Electronics 65 (11) (2018) 8828-8839.

[23] Z. Zuo, J. Song, B. Tian, M. Basin, Robust fixedtime stabilization control of generic linear systems with mismatched disturbances, IEEE Transactions on Systems, Man, and Cybernetics: Systems (2020).

[24] J. Wang, S. Li, J. Yang, B. Wu, Q. Li, Extended state observer-based sliding mode control for PWM-based DC-DC buck power converter systems with mismatched disturbances, IET Control Theory \& Applications 9 (4) (2015) 579-586.

[25] H. Zhang, G. Xiao, Y. Xie, W. Guo, C. Zhai, Tracking Differentiator Algorithms: Theories, Implementations and Applications, Vol. 717, Springer Nature, 2020.

[26] E. Moulay, W. Perruquetti, Finite time stability and stabilization of a class of continuous systems, Journal of Mathematical Analysis and Applications 323 (2) (2006) 14301443.

[27] S. P. Bhat, D. S. Bernstein, Finite-time stability of continuous autonomous systems, SIAM Journal on Control and Optimization 38 (3) (2000) 751-766.

[28] A. Levant, Higher-order sliding modes, differentiation and output-feedback control, International journal of Control 76 (9-10) (2003) 924-941.

[29] J. A. Moreno, Arbitrary order fixed-time differentiators, IEEE Transactions on Automatic Control (2021). 